\title{
Versorgungsnahe Daten zur Evaluation von Interventionseffekten: Teil 2 des Manuals
}

\section{Routine Practice Data for Evaluating Intervention Effects:} Part 2 of the Manual

\begin{abstract}
Autoren
Falk Hoffmann ${ }^{*}$, Thomas Kaiser ${ }^{2}$, Christian Apfelbacher ${ }^{3}$, Stefan Benz ${ }^{4,5}$, Thomas Bierbaum ${ }^{6}$, Karsten Dreinhöfer ${ }^{7,8}$, Michael Hauptmann ${ }^{9}$, Claus-Dieter Heidecke ${ }^{10}$, Michael Koller ${ }^{11}$, Tanja Kostuj ${ }^{12}$, Olaf Ortmannn ${ }^{13}$, Jochen Schmitt ${ }^{14}$, Holger Schünemann ${ }^{15}$, Christof Veit ${ }^{16}$, Wolfgang Hoffmann ${ }^{17}$, Monika Klinkhammer-Schalke, 18
\end{abstract}

Institute

1 Department für Versorgungsforschung, Carl von Ossietzky Universität Oldenburg, Oldenburg

2 Institut für Qualität und Wirtschaftlichkeit im Gesundheitswesen (IQWiG), Köln

3 Institut für Sozialmedizin und Gesundheitssystemforschung, Otto von Guericke Universität Magdeburg, Magdeburg

4 Kliniken Böblingen, Klinikverbund Südwest GmbH, Sindelfingen

5 Arbeitsgemeinschaft Deutscher Tumorzentren, Berlin

6 Deutsches Netzwerk Versorgungsforschung e.V., Berlin

7 Klinik für Orthopädie und Unfallchirurgie, Medical Park Berlin Humboldtmühle

8 Centrum für Muskuloskeletale Chirurgie (CMSC), Charité Universitätsmedizin Berlin

9 Institut für Biometrie und Registerforschung, Fakultät für Gesundheitswissenschaften, Medizinische Hochschule Brandenburg Theodor Fontane, Neuruppin

10 Institut für Qualitätssicherung und Transparenz im Gesundheitswesen (IQTIG), Berlin

11 Zentrum für Klinische Studien, Universitätsklinikum Regensburg, Regensburg

12 Orthopädisch-Traumatologische Zentrum, St. Marien-Hospitals Hamm, Hamm

13 Klinik für Frauenheilkunde und Geburtshilfe, Universität Regensburg Fakultät für Medizin, Regensburg

14 Zentrum für Evidenzbasierte Gesundheitsversorgung, Universitätsklinikum Carl Gustav Carus an der Technischen Universität Dresden, Dresden

15 Cochrane Canada and Mc Master GRADE Centre, WHO Collaborating Centre for Infectious Diseases, Research and Methods, Hamilton, Canada

16 BQS Institut für Qualität \& Patientensicherheit $\mathrm{GmbH}$, Hamburg

17 Institut für Community Medicine, Universität Greifswald, Greifswald
18 Tumorzentrum Regensburg, Institut für Qualitätssicherung und Versorgungsforschung, Universität Regensburg, Regensburg

\section{Schlüsselwörter}

Versorgungsforschung, Evidenzbasierte Medizin,

Real world data, Routine pratice data

Keywords

Health services research, evidence-based medicine, Real world data, Routine pratice data

online publiziert $\quad 21.05 .2021$

Bibliografie

Gesundheitswesen 2021; 83: 470-480

DOI 10.1055/a-1484-7235

ISSN $0941-3790$

(C) 2021. Thieme. All rights reserved.

Georg Thieme Verlag KG, Rüdigerstraße 14,

70469 Stuttgart, Germany

Korrespondenzadresse

Prof. Dr. Falk Hoffmann

Department für Versorgungsforschung

Carl von Ossietzky Universität Oldenburg

Ammerländer Heerstraße 140

26129 Oldenburg

Deutschland

falk.hoffmann@uol.de

\section{ZUSAMMENFASSUNG}

Die Evaluation von Interventionseffekten ist eine wichtige Domäne der Versorgungsforschung. Die vom Deutschen Netzwerk Versorgungsforschung (DNVF) ins Leben gerufene Ad hoc Kommission zur Nutzung versorgungsnaher Daten beschäftigt sich in diesem zweiten Teil des Manuals deshalb mit der Nut- 
zung dieser Daten zur Evaluation von Interventionseffekten. Zunächst erfolgt dabei eine Aufarbeitung der Begriffsbestimmung und Diskussion zur Bedeutung von Kontextfaktoren. Anschließend werden sowohl allgemeine Anforderungen an Planung, Datenerhebung und Auswertung sowie konkrete Beispiele zur Evaluation von Interventionseffekten für die 3 Anwendungsfelder Arzneimitteltherapie, nicht-medikamentöse Maßnahmen sowie komplexe Interventionen herausgearbeitet. Dabei werden sowohl Szenarien betrachtet, bei denen bisher keine Informationen aus direkt vergleichenden randomisierten kontrollierten Studien (RCTs) vorliegen bzw. bereits RCTs vorhanden sind, jedoch eine Erweiterung der Fragestellung erforderlich ist. Im Zentrum steht bei allen Beispielen an erster Stelle immer die Frage, ob die Datenquelle für die entsprechende Forschungsfrage geeignet ist und zwar unabhängig davon, ob eine Studie mit oder ohne Randomisierung durchgeführt wird. Die gewählten Beispiele stammen größtenteils aus der Onkologie, weil die hierfür notwendigen Daten zumindest in Ansätzen bereits für Deutschland vorliegen. Abschließend diskutiert das Manual mögliche Herausforderungen für eine zukünftige Nutzung dieser Daten.

\section{ABSTRACT}

The evaluation of intervention effects is an important domain of health services research. The ad hoc commission for the use of routine practice data of the German Network for Health Services Research (DNVF) therefore provides this second part of its manual focusing on the use of routine practice data for the evaluation of intervention effects. First, we discuss definition issues and the importance of contextual factors. Subsequently, general requirements for planning, data collection and analysis as well as concrete examples for the evaluation of intervention effects for the 3 fields of application regarding pharmacotherapy, nonpharmaceutical interventions as well as complex interventions are elaborated. We consider scenarios in which no information from randomized controlled trials (RCTs) comparing the two groups directly is yet available or in which RCTs are already available but an extension of the research question is required. In all examples either with or without randomization, the first and foremost question is always whether the data source is suitable for the specific research question. Most of the examples chosen are from oncology trials, because the necessary data are already available for Germany, at least in some form. Finally, the manual discusses possible challenges for future use of these data.

\section{Einleitung und Hintergrund}

Eine häufig geäußerte Kritik an randomisierten kontrollierten Studien (RCT) ist, dass sie grundsätzlich ein künstliches (,irreales“) Umfeld abbilden, insbesondere durch rigide Ein- und Ausschlusskriterien sowie häufige und aufwendige Kontrolluntersuchungen. Somit erscheine die Übertragbarkeit der Ergebnisse von RCTs auf die Versorgungsrealität fraglich. Entsprechend findet man in letzter Zeit in der Diskussion als eine Art Gegenpol häufig die Begriffe „Real World Evidence“ (RWE) und „Real World Data“ (RWD) für Ansätze, bei denen auf Daten aus Registern, elektronischen Gesundheitsakten oder andere große, teilweise direkt von Patientinnen und Patienten generierte oder unstrukturierte Datenmengen („big data“) zurückgegriffen wird [1]. Dieses Konzept suggeriert eine scheinbare Überlegenheit, weil die Daten aus der Versorgungsrealität stammen (und damit als generalisierbar oder von hoher „externer Validität“ angesehen werden), ohne dass jedoch die methodische Qualität und die Belastbarkeit der Daten bzw. des Studiendesigns zur Beantwortung der Forschungsfrage immer in gleichem Maße mitdiskutiert werden (häufig als „interne Validität“ bezeichnet). Allerdings lassen sich nur aus Erkenntnissen methodisch hochwertiger Studien versorgungsrelevante Entscheidungen treffen. Der Kontextbezug solcher Studien ist darüber hinaus zu prüfen. Die Nutzbarkeit solcher aus der Versorgung stammenden Daten zur Beantwortung der jeweiligen Forschungsfrage muss insgesamt sorgfältig geprüft und transparent dargestellt werden.

Diese wissenschaftliche und methodische Diskussion wird aktuell immer wichtiger, da internationale Entwicklungen der letzten Jahre die zunehmende Relevanz solcher Daten z.B. für die Zulassung von Arzneimitteln zeigen (z.B. 2017 im Rahmen des „21st Century Cures Act“ durch die amerikanische Food and Drug Admi- nistration (FDA) [2] oder 2020 in dem Entwurf einer Guideline der europäischen Zulassungsbehörde EMA zu Registerstudien [3]). Auch auf nationaler Ebene wurde 2020 zu diesem Thema durch das Institut für Qualität und Wirtschaftlichkeit im Gesundheitswesen (IQWiG) ein Rapid Report „Konzepte zur Generierung versorgungsnaher Daten und deren Auswertung zum Zwecke der Nutzenbewertung von Arzneimitteln nach § 35a SGB V“ erstellt [4]. Gleichzeitig bietet die in den letzten Jahren fortschreitende Digitalisierung im Gesundheitswesen die große Chance, eine hohe Informationsbreite bei einem vertretbaren Aufwand in der Datenerfassung zu erreichen.

Um den weiteren wissenschaftlichen Diskurs zur Nutzbarkeit dieser Daten voranzubringen, hat das Deutsche Netzwerk Versorgungsforschung (DNVF) Anfang 2020 eine Ad hoc Kommission gegründet, deren Mitglieder die Autorinnen und Autoren dieses Beitrags sind. Die Ad hoc Kommission hat sich bewusst gegen die Verwendung der Begriffe „Real World Evidence“ (RWE) und „Real World Data“ (RWD) entschieden, da manche Autoren und Institutionen damit eine scheinbare Überlegenheit gegenüber RCTs suggerieren oder RCTs auf Basis von Daten aus der Versorgung sogar ausschließen. Auch RCTs können jedoch unter wenig einschränkenden Bedingungen und mit Rückgriff auf Daten aus der Routineversorgung durchgeführt werden [5, 6]. Von der Ad hoc Kommission wurde stattdessen der eher inhaltliche Begriff der „versorgungsnahen Daten“ (VeDa) gewählt, da letztlich jede Art von empirischer, wissenschaftlich fundierter Evidenz real ist („all evidence is real world evidence“ [7]). Dieser Begriff wird auch im IQWiG Rapid Report verwendet [4]. Insgesamt muss es darum gehen, die beste Evidenz für die jeweilige Fragestellung zu definieren, zu identifizieren und in die Entscheidungsfindung zu integrieren. Dies schließt je nach 
Fragestellung auch Evidenz, die sich mit Patientenwerten, Akzeptanz und Machbarkeit befasst, mit ein.

In der Ad hoc Kommission wurde beschlossen, das Thema als Manual mit mehreren Teilen zu bearbeiten und zu publizieren. Bereits im September 2020 erschien der erste Teil „Manual für Methoden und Nutzung versorgungsnaher Daten zur Wissensgenerierung “ in dieser Zeitschrift [8]. In diesem Manual wurde neben der Begriffsbestimmung auch die internationale sowie nationale Bedeutung der Forschung mit VeDa vertiefend dargestellt. Untermauert mit zahlreichen Beispielen, wurden zudem die beiden Themenbereiche Evaluation von Interventionseffekten sowie Versorgungsanalysen für die Nutzung von VeDa herausgearbeitet.

Ziel dieser zweiten Publikation der Ad hoc Kommission ist es, Rahmenbedingungen für die Anwendung von VeDa zur Evaluation von Interventionseffekten zu skizzieren.

\section{Interventionen im komplexen Kontext}

Die Evaluation von Interventionseffekten ist eine wichtige Domäne der Versorgungsforschung. Dies wird u.a. dadurch deutlich, dass der Innovationsfonds seit 2016 Projekte aus den Bereichen Versorgungsforschung und neue Versorgungsformen mit einem hohen Finanzierungsvolumen fördert und als eine wesentliche Voraussetzung für die Förderung ein tragfähiges Evaluationskonzept fordert. Der Innovationsfonds legt sich dabei nicht auf ein spezifisches Studiendesign fest. Dies gilt auch für den Rapid Report des IQWiG, der nicht das Design, sondern vor allem die Datenquellen und die Ergebnissicherheit der daraus zu gewinnenden Evidenz in den Vordergrund stellt [4].

Eine bedeutsame Herausforderung von Studien zur Evaluation von Interventionseffekten in der Versorgungsforschung ist der Umgang mit Komplexität. Dies betrifft sowohl die Komplexität der Intervention selbst als auch des Kontextes (z.B. in Bezug auf geografische Lage, Setting, Patientencharakteristika und beteiligte Berufsgruppen), in dem die Intervention implementiert bzw. evaluiert werden soll (sog. „doppelte Komplexität“) [9]. Sowohl der Kontext als auch die Intervention können zudem aus mehreren nicht isolierbaren Einzelfaktoren bzw. Ebenen bestehen, die miteinander in Wechselwirkungen stehen. Soll bspw. die Wirksamkeit einer interdisziplinären Fallbesprechung zwischen Pflegekräften, Hausärztinnen und Hausärzten sowie Apothekerinnen und Apothekern im Pflegeheim untersucht werden, können sich daraus zahlreiche Wechselwirkungen ergeben, die bei der Planung, Durchführung und Auswertung einer entsprechenden Studie zu berücksichtigen sind [10].

Kontextfaktoren spielen zum einen auf der Studienebene eine Rolle, z.B. bei der Frage, ob eine komplexe Intervention mit ihren Einzelkomponenten von einer Situation auf eine andere übertragen werden kann. Beispielsweise muss auf Studienebene beachtet werden, ob die untersuchte komplexe Intervention (z.B. ein bestimmtes Rehabilitationsverfahren) mit ihren in der Studie untersuchten Einzelkomponenten in der Praxis tatsächlich so durchgeführt werden kann, weil möglicherweise die Einzelkomponenten wie psychologische Betreuung nicht überall vorhanden sind [11]. Zum anderen sind Kontextfaktoren auf der Ebene der Entscheidungsfindung relevant, z.B. auf der Systemebene, bei der Kriterien wie Kosten, Akzeptanz und Machbarkeit (lässt sich die komplexe Intervention implementieren?) eine Rolle spielen [11].

\section{Allgemeine Anforderungen an Planung, Datenerhebung und Auswertung}

Die hier skizzierten Anforderungen und Empfehlungen bei der Nutzung von VeDa zur Evaluation von Interventionseffekten folgen dem in der ersten Publikation herausgearbeiteten strukturierten Vorgehen entsprechend der Prozessschritte Fragestellung, Planung, Datenerhebung, Auswertung, Interpretation und Bewertung sowie Umsetzung in der Versorgung [8].

Anhand dieser Prozessschritte werden in $>$ Tab. 1 wesentliche Eckpunkte zum Vorgehen bei der Evaluation von Interventionseffekten auf Basis von VeDa dargestellt, und zwar für die drei Anwendungsfelder Arzneimitteltherapie, nicht-medikamentöse Maßnahmen sowie komplexe Interventionen. Eine Trennung der beiden letztgenannten Anwendungsfelder erfolgt bewusst, da bei der Evaluation nicht-medikamentöser Verfahren (z.B. dem Vergleich von verschiedenen Kniegelenkendoprothesen oder von verschiedenen psychotherapeutischen Verfahren) teilweise andere Aspekte zu berücksichtigen sind als bei noch komplexeren sektoren- und disziplinenübergreifenden Fragestellungen. Beispielhaft steht hierfür die Wirksamkeit einer telemedizinischen Anwendung bei Herzinsuffizienz oder das oben skizzierte Beispiel der interdisziplinären Fallbesprechungen. Entsprechend bilden diese drei Anwendungsfelder die Spannbreite von Interventionen und Kontextfaktoren mit unterschiedlicher Komplexität innerhalb der Versorgungsforschung ab.

- Tab. 1 zeigt ebenfalls, dass Grundprinzipien der Studienplanung, der Datenerhebung und der Auswertung für alle Anwendungsfelder in gleichem Maße gelten, und die Prozessschritte unabhängig vom Themenfeld nur aufeinander aufbauend zu betrachten sind. Die Formulierung der Fragestellung ist die Grundlage für die Auswahl des passenden Studiendesigns und der für die Beantwortung der Fragestellung notwendigen Daten. Im Fokus des folgenden Textes stehen die beiden Aspekte Studiendesign und Daten.

\section{Kriterien zur Beurteilung der Eignung und Qualität der Daten}

In > Tab. 2 sind die Kriterien der Eignung und Qualität der Datenerhebung beispielhaft für Registerstudien zur Evaluation von Interventionseffekten dargestellt. Diese Kriterien sind prinzipiell für alle genannten Anwendungsfelder und andere Datenquellen (z.B. elektronische Patientenakten) anwendbar, ggf. sind sie um themenspezifische Aspekte zu ergänzen.

Diese Kriterienliste ermöglicht zum einen eine orientierende Prüfung, ob eine spezifische Datenquelle prinzipiell für Studien zur Evaluation von Interventionseffekten geeignet ist. Sie kann zum anderen Betreibern von Datenquellen (z.B. Indikationsregistern) als Unterstützung dienen, um die Datenquelle auf die zukünftige Durchführung solcher Studien vorzubereiten.

Bei der Kriterienliste kann zwischen übergeordneten Kriterien (grundsätzliche Qualität der Datenquelle) und fragestellungsbezogenen Kriterien (Notwendigkeit der Erhebung spezifischer Daten) unterschieden werden. Wichtig ist, dass die Eignung einer Datenquelle als Grundlage für eine Studie zur Evaluation von Interventionseffekten immer fragestellungsbezogen zu prüfen ist. Dieselbe Datenquelle kann für eine Fragestellung geeignet sein (z.B. zur 
> Tab. 1 Prozessschritte beim Einsatz versorgungsnaher Daten zur Evaluation von Interventionseffekten in verschiedenen Anwendungsfeldern.

\begin{tabular}{|c|c|c|c|}
\hline Prozessschritt & Arzneimitteltherapie & $\begin{array}{l}\text { Nicht-medikamentöse } \\
\text { Verfahren }\end{array}$ & Komplexe Interventionen \\
\hline Fragstellung & \multicolumn{3}{|c|}{$\begin{array}{l}\text { - Identifikation einer offenen Fragestellung aus der Versorgung (Evidenzlücke) } \\
\text { - Formulierung der Fragestellung gemäß PICO-Schema }\end{array}$} \\
\hline \multirow[t]{2}{*}{ Planung } & \multicolumn{3}{|c|}{$\begin{array}{l}\text { - Auswahl des Studiendesigns unter Berücksichtigung der erwarteten Therapieeffekte, der Machbarkeit und bereits vorliegenc } \\
\text { Evidenz } \\
\text { - Primär: Durchführbarkeit einer versorgungsnahen RCT (z.B. registerbasierte RCT) prüfen } \\
\text { - Bei Studien mit oder ohne Randomisierung: Studienplanung gemäß Behandlungsalltag (Ein- und Ausschlusskriterien, } \\
\text { Interventionen, Endpunkte, Visiten); Ausnahmen sind Festlegungen, die sich aus der PICO-Fragestellung ergeben (z.B. } \\
\text { genaue Definition der Intervention, Erhebung der relevanten Endpunkte) } \\
\text { - Bei Studien ohne Randomisierung } \\
\text { - Strukturierte Planung einer wünschenswerten, fiktiven RCT (z.B. mit Hilfe des Target Trial Konzepts) } \\
\text { - Systematische Identifikation und Präspezifikation möglicher Confounder } \\
\text { - Durchführung der Studie ohne Randomisierung nur bei grundsätzlicher Eignung der Datenquelle (Validität der Daten: } \\
\text { Basisdaten, Verlaufsdaten, Erhebungszeitpunkte, Endpunkte, Confounder usw.) } \\
\text { - Vor Beginn der Datenerhebung finalisiertes Studienprotokoll (bei retrospektiven Analysen: vor Beginn der Auswertung) }\end{array}$} \\
\hline & $\begin{array}{l}\text { - Prüfen, ob aufgrund } \\
\text { der Fragestellung } \\
\text { die Arzneimittel- } \\
\text { therapie gemäß } \\
\text { Zulassung erfolgen } \\
\text { muss (bei } \\
\text { interventionellen } \\
\text { Studien: Vorgabe im } \\
\text { Studienprotokoll; } \\
\text { bei nicht interven- } \\
\text { tionellen Studien: } \\
\text { Beschränkung auf } \\
\text { entsprechende } \\
\text { Datensätze) }\end{array}$ & $\begin{array}{l}\text { - Bei Verfahren, die nicht } \\
\text { häufig angewendet werden, } \\
\text { exakte Definition dieser } \\
\text { Verfahren (bei interventio- } \\
\text { nellen Studien: Vorgabe im } \\
\text { Studienprotokoll; bei nicht } \\
\text { interventionellen Studien: } \\
\text { Beschränkung auf } \\
\text { entsprechende Datensätze) } \\
\text { - Prüfen, ob die Zuordnung } \\
\text { zum Verfahren an „Schulen“ } \\
\text { und nicht an prognostische } \\
\text { Merkmale gebunden ist } \\
\text { (instrumental variable [35]) }\end{array}$ & $\begin{array}{l}\text { - Intervention, Setting und Populationen genau definieren } \\
\text { („System, auf das die Intervention wirken soll“) } \\
\text { - Partizipative Elemente sind konstitutiv (Patienten, Leistungs- } \\
\text { erbringer, Aufsichtsbehörden, weitere Stakeholder) } \\
\text { - Pilotierung von Designelementen i.d.R. unverzichtbar } \\
\text { (Präzisierung der Intervention, Rekrutierung und Endpunkerhe- } \\
\text { bung) } \\
\text { - Prozessevaluation und -monitoring berücksichtigen (u.a. } \\
\text { Rekrutierungserfolg, Reaktion des Systems auf Intervention, } \\
\text { Ermittlung förderlicher und hindernder Faktoren, Identifikation } \\
\text { von Determinanten der Effekte auf die Endpunkte) } \\
\text { - RCT: Häufig Cluster-Randomisierung (i.d.R. auf Ebene der } \\
\text { Institution), aber auch individuelle Randomisierung möglich } \\
\text { - Es kann überlegt werden, auf eine detaillierte Baseline-Erhebung } \\
\text { zu verzichten (weil bereits das i.d.R. eine Intervention darstellt) } \\
\text { - Studien ohne Randomisierung: } \\
\text { Bei Wahl der Kontrolle auf Ähnlichkeit möglichst vieler } \\
\text { Randbedingungen achten (eher globale Auswahl, z.B. } \\
\text { vergleichbarer Landkreis o.ä.) } \\
\text { - Basiserhebung wichtiger als bei RCT }\end{array}$ \\
\hline \multirow[t]{2}{*}{ Datenerhebung } & \multicolumn{3}{|c|}{$\begin{array}{l}\text { - Gewähltes Instrument zur Datenerhebung / spezifische Datenstruktur (z.B. Register) muss Daten in der notwendigen Qualität } \\
\text { zur Verfügung stellen können (inkl. Daten zu Begleit- und Folgeinterventionen, bei Studien ohne Randomisierung auch inkl. } \\
\text { Daten für Confounderkontrolle) } \\
\text { - Möglichst kontinuierlicher Datenfluss - insbesondere bei geplanten Zwischenauswertungen } \\
\text { - Möglichst Onlinedatenerhebung und Plausibilitätsregeln hinterlegen sowie Datenübermittlung / Speicherung nur bei vollständi- } \\
\text { gen (Teil)Datensätzen zulassen } \\
\text { - Bei interventionellen Studien mit oder ohne Randomisierung: Nutzung bestehender Strukturen zur Erhebung der VeDa prüfen } \\
\text { (z.B. Indikationsregister) }\end{array}$} \\
\hline & & & $\begin{array}{l}\text { Generell: } \\
\text { - Kombination aus systembezogenen und individuellen } \\
\text { Endpunkten bevorzugen (vorab sowohl präzise als auch } \\
\text { unmittelbar umsetzbar definieren) } \\
\text { - i.a.R. sind Mixed-Methods-Ansätze mit qualitativen und } \\
\text { quantitativen Methoden erforderlich, jedoch in ausgewogenem } \\
\text { Maß } \\
\text { - Berücksichtigen, dass sowohl Datenerhebung als auch } \\
\text { Prozessmonitoring einen Einfluss auf die Durchführung und den } \\
\text { Effekt der Intervention haben kann } \\
\text { - Erhebung der Endpunkte nicht durch Interventionspersonal } \\
\text { - RCT: Bei Cluster-Randomisierung Start der Intervention erst } \\
\text { nach Baseline-Erhebung }\end{array}$ \\
\hline Auswertung & \multicolumn{3}{|c|}{$\begin{array}{l}\text { - Vor Datenbankschluss und vor Beginn der Auswertung finalisierter statistischer Analyseplan (SAP); Auswertung gemäß des } \\
\text { präspezifizierten SAPs } \\
\text { - Bei Studien ohne Randomisierung: präspezifizierter Algorithmus zur Confounderkontrolle und Adjustierung in der Analyse; } \\
\text { Definition von Abbruchkriterien (z.B. keine ausreichende Balanciertheit der Daten trotz Adjustierung zu erreichen) } \\
\text { - etwaige geplante Zwischenanalysen in der Methodik berücksichtigen (z.B. p-Wert anpassen) }\end{array}$} \\
\hline
\end{tabular}


Tab. 1 Fortsetzung.

\begin{tabular}{|c|c|c|c|}
\hline Prozessschritt & Arzneimitteltherapie & $\begin{array}{l}\text { Nicht-medikamentöse } \\
\text { Verfahren }\end{array}$ & Komplexe Interventionen \\
\hline $\begin{array}{l}\text { Interpretation } \\
\text { und Bewertung }\end{array}$ & \multicolumn{3}{|c|}{$\begin{array}{l}\text { - Berücksichtigung der Aussagekraft der unterschiedlichen Studiendesigns und der konkreten Datenqualität bei der Interpretation } \\
\text { der Ergebnisse } \\
\text { - Bei Studien ohne Randomisierung: Ableitung von Aussagen zum Nutzen oder Schaden in Abhängigkeit von der Effektstärke und } \\
\text { den Confoundern } \\
\text { - in der Regel nur bei ausreichend großen Therapieeffekten (z. B. RR < 0,5) } \\
\text { - ggf. bei Übertragbarkeitsfragestellungen unter Verwendung bereits vorhandener RCTs auch bei kleineren Effekten } \\
\text { - wenn alle plausiblen Confounder und anderen Effektverzerrer eine entgegengesetzte Aussage vorschlagen } \\
\text { - Kritische Interpretation etwaig genutzter Sekundärdaten } \\
\text { - Kritische Diskussion der Übertragbarkeit auf ähnliche Settings, Regionen, Patientengruppen usw. }\end{array}$} \\
\hline Publikation & \multicolumn{3}{|c|}{$\begin{array}{l}\text { - Erstellung eines Ergebnisberichts gemäß ICH E3 } \\
\text { - Erstellung einer wissenschaftlichen Publikation mit begleitender Veröffentlichung des vollständigen Ergebnisberichts inklusive } \\
\text { Studienprotokoll und SAP }\end{array}$} \\
\hline $\begin{array}{l}\text { Ableitung von } \\
\text { Empfehlungen } \\
\text { und Umsetzung }\end{array}$ & \multicolumn{3}{|c|}{$\begin{array}{l}\text { - Beurteilung im Gesamtkontext der Evidenz } \\
\text { - Sicherstellung der Durchdringung der Ergebnisse in der Fachwelt / bei den Versorgern } \\
\text { - Berücksichtigung des Einflusses relevanter Kontextfaktoren } \\
\text { - Nutzung der identifizierten hindernden und förderlichen Faktoren } \\
\text { - Rekrutierungsprobleme berücksichtigen }\end{array}$} \\
\hline
\end{tabular}

> Tab. 2 Kriterien der Eignung und Qualität der Datenerhebung für Registerstudien zur Evaluation von Interventionseffekten (aus [4]).

\begin{tabular}{|c|c|}
\hline Kategorie & Qualitätskriterien \\
\hline $\begin{array}{l}\text { Obligatorische Kriterien zur } \\
\text { Sicherstellung der Datenqualität }\end{array}$ & $\begin{array}{l}\text { - Detaillierte Registerbeschreibung (Zielsetzung, Registerprotokoll) } \\
\text { - Exakte Definition / Operationalisierung von Expositionen, klinischen Ereignissen, Endpunkten und } \\
\text { - } \text { Aktueller Datenplan / Kodierhandbuch } \\
\text { - Schulungen zur Datenerhebung und -erfassung } \\
\text { - Klar definierte Ein- und Ausschlusskriterien für Registerpatienten } \\
\text { - SOP-System zur Datenerhebung } \\
\text { - Maßnahmenpaket zur Sicherstellung der Richtigkeit der Daten und zur Information über Fehlerraten } \\
\text { (z. B. source data verification, interne und externe Audits, IT-gestützte Prüfungen [z. B. Cross-Refe- } \\
\text { - } \text { - Audit trail - Dokumentation der Prozess- und Definitionsänderungen im Register } \\
\text { - Wissenschaftliche Unabhängigkeit und Neutralität } \\
\text { - Nachhaltige Finanzierung }\end{array}$ \\
\hline $\begin{array}{l}\text { Allgemeine Kriterien, die regelhaft für } \\
\text { Registerstudien zum Zwecke der } \\
\text { Nutzenbewertung von Arzneimitteln } \\
\text { relevant sind }\end{array}$ & $\begin{array}{l}\text { - Verwendung exakter Datumsangaben zum Patienten, zur Erkrankung und zu Ereignissen } \\
\text { - Detaillierte Informationen zur Intervention (z.B. bei Arzneimitteltherapie Wirkstoff, Dosis, Dosisände- } \\
\text { - Tung, inkl. Datumsangaben) } \\
\text { - Timeliness (Aktualität / schnelle Verfügbarkeit / Pünktlichkeit der benötigten Ergebnisse) }\end{array}$ \\
\hline $\begin{array}{l}\text { Allgemeine Kriterien, die je nach } \\
\text { Fragestellung für Registerstudien zum } \\
\text { Zwecke der Nutzenbewertung relevant } \\
\text { sein können }\end{array}$ & $\begin{array}{l}\text { - Verwendung von Standard-Klassifikationen (z. B. ICD-10) und Terminologien (z. B. MedDRA) } \\
\text { - Verwendung valider Standard-Erhebungsinstrumente (Fragebogen, Skalen, Tests) } \\
\text { - Flexibilität und Anpassungsfähigkeit (z. B. zur Einbettung von Studien, für weitere Datenerhebung, bei } \\
\text { veränderter Versorgungssituation) } \\
\text { - Verknüpfbarkeit mit anderen Datenquellen }\end{array}$ \\
\hline $\begin{array}{l}\text { Kriterien, deren Erfüllungsgrad } \\
\text { fragestellungsbezogen zu beurteilen ist }\end{array}$ & $\begin{array}{l}\text { - Repräsentativität der Stichprobe / Selektion der Stichprobe } \\
\text { - Vollständigkeit der Daten je Erhebungszeitpunkt (loss-to-follow-up, Drop-outs) } \\
\text { - Vollständigkeit der Erhebungszeitpunkte } \\
\text { - Richtigkeit der Daten } \\
\text { - Erhebung aller für die Fragestellung relevanten Confounder } \\
\text { - Datenkonsistenz über die Zeit }\end{array}$ \\
\hline \multicolumn{2}{|c|}{$\begin{array}{l}\text { aDie genannten Kriterien sind wichtige Kriterien der Datenqualität, jedoch nur fragestellungsbezogen zu beurteilen. Zum einen beziehen sich z. B. } \\
\text { „Richtigkeit der Daten“ und „Datenkonsistenz über die Zeit“ nur auf die Daten, die für die jeweilige Fragestellung relevant sind. Zum anderen bezieht } \\
\text { sich „Repräsentativität der Stichprobe“ nur auf die für die Fragestellung relevante Population, nicht jedoch auf die gesamte Registerpopulation. }\end{array}$} \\
\hline
\end{tabular}

Untersuchung von Effekten auf das Gesamtüberleben), für eine andere hingegen nicht (z.B. zur Untersuchung von Effekten auf die gesundheitsbezogene Lebensqualität, beispielsweise weil diese in der Datenquelle nicht erhoben wird). Die Gesamtqualität der Daten oder Evidenz kann demnach auch für verschiedene Endpunkte unterschiedlich sein. 
- Tab. 3 Vergleich eines Studienprotokolls einer RCT und einer Studie auf Basis von VeDa mittels Target Trial Emulation anhand eines Beispiels (nach $[14,15])$

\begin{tabular}{|c|c|c|}
\hline Protokollkomponente & RCT (Target Trial) & Studie mit VeDa (Target Trial Emulation) \\
\hline Ziel & $\begin{array}{l}\text { - Effekt einer Hormonersatztherapie bei postmenopausalen } \\
\text { Frauen auf das Brustkrebsrisiko innerhalb von } 5 \text { Jahren }\end{array}$ & - Identisch \\
\hline Einschlusskriterien & $\begin{array}{l}\text { - Frauen mit Menopause seit mind. } 5 \text { Jahren } \\
\text { - Keine Tumordiagnose } \\
\text { - Keine Hormonersatztherapie in den letzten } 2 \text { Jahren }\end{array}$ & $\begin{array}{l}\text { - Identisch (Validität der Operationalisierung muss } \\
\text { gewährleistet sein) }\end{array}$ \\
\hline Interventionen & $\begin{array}{l}\text { - A. Begin einer Hormonersatztherapie zu Baseline } \\
\text { (Therapieabbruch bei tiefer Beinvenenthrombose, } \\
\text { Lungenembolie, Myokardinfarkt) } \\
\text { - B. Kein Beginn Hormonersatztherapie zu Baseline }\end{array}$ & - Identisch \\
\hline Zuteilung & $\begin{array}{l}\text { - Randomisierung zu Baseline, und Patientinnen sind nicht } \\
\text { verblindet }\end{array}$ & $\begin{array}{l}\text { - Patientinnen werden zur Interventionsgruppe zugeteilt } \\
\text { bei Therapiebeginn; Zuteilung zur Kontrollgruppe } \\
\text { analog zum Zeitpunkt der Therapieentscheidung } \\
\text { - Nachbildung der Randomisierung durch umfassende } \\
\text { Adjustierung für Baselinecharakteristika (alle } \\
\text { relevanten Counfounder müssen vorliegen) }\end{array}$ \\
\hline Follow-Up & $\begin{array}{l}\text { - Beginnt mit Randomisierung und endet mit gesicherter } \\
\text { Brustkrebsdiagnose, Tod, Lost to follow-up oder } 5 \text { Jahre } \\
\text { nach Baseline (je nach dem, was zuerst kommt) }\end{array}$ & $\begin{array}{l}\text { - Identisch (besondere Herausforderung bei Beobach- } \\
\text { tungsstudien ist der Beginn des Follow-Up in der } \\
\text { Gruppe ohne Therapie) }\end{array}$ \\
\hline Endpunkt & $\begin{array}{l}\text { - Durch Biopsie gesicherte Brustkrebsdiagnose innerhalb } \\
\text { von } 5 \text { Jahren nach Randomisierung }\end{array}$ & $\begin{array}{l}\text { - Identisch (Validität der Operationalisierung muss } \\
\text { gewährleistet sein) }\end{array}$ \\
\hline $\begin{array}{l}\text { Effekt zwischen } \\
\text { Interventionen („,causal } \\
\text { contrast“) und Analyse }\end{array}$ & $\begin{array}{l}\text { - Effekt einer Zuteilung zur Hormonersatztherapie zu } \\
\text { Baseline vs. keine Hormonersatztherapie (Confounder- } \\
\text { Adjustierung optional) }\end{array}$ & $\begin{array}{l}\text { - Abweichend: Effekt eines Beginns der Hormonersatz- } \\
\text { therapie vs. keine Hormonersatztherapie (Confounder- } \\
\text { Adjustierung obligat) }\end{array}$ \\
\hline
\end{tabular}

\section{Allgemeine Methodik zur Evaluation von Interventionseffekten mit VeDa}

Für alle Anwendungsfelder adressiert die interessierende Fragestellung einen Vergleich zweier Interventionen A vs. B, wobei A die zu untersuchende Intervention und B sowohl eine andere Intervention als auch die Regelversorgung („usual care“) sein kann. Für die Evaluation von Interventionseffekten sind daher grundsätzlich vergleichende Studien erforderlich [4].Vergleichende Studien können prinzipiell mit oder ohne Randomisierung durchgeführt werden. Wenn immer möglich, sollten für die Evaluation von Interventionseffekten aus den vielfach beschriebenen Gründen RCTs herangezogen oder durchgeführt werden, und dies gilt auch bei der Verwendung von VeDa $[12,13]$.

Wenn eine Randomisierung nicht durchführbar ist oder geeignet erscheint (z.B. wegen der Notwendigkeit einer zeitnahen ersten Einschätzung auf Basis bereits erhobener Daten) sollte der geplante nicht-randomisierte Vergleich konzeptionell dennoch weitgehend wie eine RCT geplant werden. Für ein strukturiertes Vorgehen ist hierfür das z.B. in Hernan 2016 beschriebene Target Trial Konzept hilfreich [14].

Dieses Konzept zielt darauf ab, die „Grundprinzipien“ der Planung einer wünschenswerten, fiktiven RCT (des sogenannten Taget Trials) auf Basis von Beobachtungsstudien nachzubilden (die sogenannte Target Trial Emulation) [14-16]. Dadurch werden die notwendigen Protokollkomponenten, z.B. zu Ein- und Ausschlusskriterien, Endpunkte und Follow-Up, der fiktiven RCT auf die vorhandene Datenquelle übertragen. Damit kann überprüft werden, ob die Datenquelle die erforderlichen Daten z.B. zur Beschreibung der Population, zu Endpunkten, zu Erhebungszeitpunkten, zu Confoundern etc. im notwendigen Umfang und mit ausreichender Validität enthält. Ist dies der Fall, ist die Datenquelle zunächst prinzipiell für die Durchführung einer Studie ohne Randomisierung zum Vergleich A vs. B geeignet. Das Target Trial Konzept wird in $>$ Tab. 3 beispielhaft anhand einer Studie zum Effekt einer Hormonersatztherapie bei postmenopausalen Frauen auf das Brustkrebsrisiko innerhalb von 5 Jahren illustriert.

\section{Auswahl des Studiendesigns in Abhängigkeit von der Datenlage}

Bei einer offenen Fragestellung A vs. B sind konzeptionell zum Zeitpunkt der Studienplanung insbesondere zwei unterschiedliche Ausgangsszenarien denkbar:

1. Es liegt bislang keine Information aus direkt vergleichenden RCTs zum Vergleich A vs. B vor (neue Fragestellung).

2. Es liegen eine oder mehrere RCTs zum Vergleich A vs. B vor, allerdings fehlen wichtige Informationen z. B. zu Kindern und Jugendlichen, Patientinnen und Patienten höheren Alters oder solchen mit Komorbiditäten, zu seltenen Risiken, Langzeiteffekten etc. Im Bereich komplexer Interventionen kann es auch möglich sein, dass zur Wirksamkeit einzelner Elemente (oder deren Kombination) bereits RCTs vorliegen (Übertragbarkeitsfragestellung).

Im ersten Szenario (neue Fragestellung) sollte möglichst ein RCT mit VeDa (z. B. registerbasierte RCT) geplant werden. In dieser Situation wäre eine alleinige Evidenz aus Studien ohne Randomisierung mit einer sehr hohen Ergebnisunsicherheit behaftet und insbesondere für Entscheidungen mit Konsequenzen für das Gesundheitssystem unzureichend. Die aktuellen Erfahrungen zu COVID-19 zeigen eindrucksvoll, dass versorgungsnahe, pragmatische RCTs offenbar eine effiziente und gut realisierbare Methode zur Evidenzgenerierung in solchen Fällen darstellen können $[17,18]$. Sollte 
dennoch eine Studie ohne Randomisierung durchgeführt werden, z.B. weil zeitnah auf Basis bereits erhobener Daten eine erste Einschätzung erforderlich ist, sollte das oben beschriebene Target Trial Konzept für die strukturierte Studienplanung verwendet werden.

Im zweiten Szenario (Übertragbarkeitsfragestellung) könnte unter Verwendung der Erkenntnisse aus den bereits vorhandenen RCTs in einem zweischrittigen Verfahren Evidenz aus vergleichenden Studien ohne Randomisierung zur Adressierung der Informationslücken herangezogen werden:

- Im ersten Schritt wird geprüft, ob die Datenquelle (z.B. Register) zur Adressierung der Informationslücken prinzipiell geeignet ist, und es wird die Ergebnissicherheit der Studie ohne Randomisierung in dieser Datenquelle eingeschätzt. Hierfür sollte auf Basis der bereits vorhandenen RCT(s) und mithilfe des oben beschriebenen Target Trial Konzepts eine Target Trial Emulation zur Anwendung kommen [14]. Mit der daraus resultierenden nicht randomisierten Studie kann zum einen geprüft werden, ob die Datenquelle prinzipiell für die Durchführung einer Studie zum Vergleich A vs. B geeignet ist. Zum anderen kann die Ergebnissicherheit dieser nicht randomisierten Studie abgeschätzt werden, indem die Effekte zwischen der Target Trial Emulation mit denen aus den bereits vorhandenen RCTs abgeglichen werden. Bei einer ausreichenden Ergebnissicherheit ist ein Vergleich ohne Randomisierung in dieser Datenquelle sinnvoll.

- Im zweiten Schritt wird, sofern gemäß Schritt 1 die Datenquelle geeignet und die zu erwartende Ergebnissicherheit ausreichend ist, die eigentliche vergleichende Studie ohne Randomisierung durchgeführt. Auch hierbei sollte das Target Trial Konzept verwendet werden, und die Target Trial Emulation entspricht der aus dem ersten Schritt mit Anpassung der Aspekte, für die die Informationslücke besteht (z.B. Einschluss anderer Altersgruppen, längere Beobachtungsdauer). Je nach Datengrundlage kann dieser Schritt prospektiv oder unter Verwendung bereits erhobener Daten erfolgen.

Grundsätzlich wäre für beide Szenarien auch denkbar, einen adjustierten indirekten Vergleich durchzuführen, falls RCTs zu A und B im Vergleich mit anderen Komparatoren vorliegen [19, 20]. Allerdings haben solche indirekten Vergleiche zum einen eine geringere Ergebnissicherheit als direkt vergleichende, pragmatische RCTs (Szenario 1). Zum anderen wäre es für die in Szenario 2 beschriebene Ausgangssituation notwendig, dass die im indirekten Vergleich einzuschließenden RCTs gerade für diejenigen Patientengruppen vorliegen, für die bislang keine direkt vergleichenden RCTs durchgeführt wurden (z.B. Kinder oder ältere Patientinnen und Patienten). Schließlich hängt die Durchführung indirekter Vergleiche grundsätzlich davon ab, ob ausreichend ähnliche Studien vorliegen und die Daten zu diesen Studien auch zur Verfügung stehen [21].

\section{Beispiele für die Anwendungsfelder}

Für die 3 Anwendungsfelder Arzneimitteltherapie, nicht-medikamentöse Maßnahmen und komplexe Interventionen wird nachfolgend anhand von Beispielen das oben beschriebene konzeptionelle Vorgehen illustriert.

\section{Anwendungsfeld Arzneimitteltherapie}

Beispiel 1

Für die Behandlung des fortgeschrittenen Mammakarzinoms wurden seit 20163 verschiedene Wirkstoffe aus der Wirkstoffgruppe der CDK4/6-Inhibitoren zur Kombinationstherapie mit einem Aromatasehemmer zugelassen. Die Ergebnisse der zugehörigen Nutzenbewertungen fielen unterschiedlich aus (von „kein Zusatznutzen“ bis „geringer Zusatznutzen“) [22-24]. Dabei unterschied sich neben den Ergebnissen zum Gesamtüberleben auch die Datenlage zu patientenberichteten Endpunkten (PRO wie Symptome, gesundheitsbezogene Lebensqualität). Allen 3 Wirkstoffen gemeinsam ist, dass keine aussagekräftige PRO-Daten für Patientinnen nach Krankheitsprogression vorliegen. RCTs zum direkten Vergleich der 3 Wirkstoffe fehlen. Insgesamt bleibt unklar, ob einer der 3 Wirkstoffe bevorzugt eingesetzt werden sollte. Diese Informationslücke könnte durch eine pragmatische, registerbasierte RCT adressiert werden, die versorgungsnah und über eine etwaige Krankheitsprogression hinaus Daten zu Symptomen und zur Lebensqualität der Patientinnen erhebt.

\section{Beispiel 2}

In der Nutzenbewertung des Wirkstoffs Sarilumab zur Behandlung der Rheumatoiden Arthritis wurde aufgrund der Zulassungsstudie für Patientinnen und Patienten mit erstmaliger Biologika-Therapie ein beträchtlicher Zusatznutzen gegenüber Adalimumab festgestellt [25]. Die Ergebnissicherheit war jedoch gering, unter anderem, weil Daten zur wichtigen Gruppe der Patientinnen und Patienten mit einer Kontraindikation gegenüber Methotrexat (MTX) fehlten. Darüber hinaus lagen nur Daten für einen Zeitraum von 24 Wochen vor.

In diesem Fall könnte in einem ersten Schritt ein nicht randomisierter Vergleich als Nachbildung der Zulassungsstudie durchgeführt werden, d.h. unter Ausschluss von Patientinnen und Patienten mit MTX-Kontraindikationen, aber ohne Randomisierung. Dabei könnte zur Unterstützung eines strukturierten Vorgehens das Target Trial Konzept verwendet werden. Als Datenquelle könnte z.B. ein vorhandenes Indikationsregister (z.B. das RABBIT-Register [26]) dienen. Lässt sich dadurch zum einen die Zulassungsstudie nachbilden (prinzipielle Eignung der Datenquelle) und sind zum anderen die Effekte des resultierenden nicht randomisierten Vergleichs ähnlich zu denen in der randomisierten Zulassungsstudie (Voraussetzung für ausreichende Ergebnissicherheit des nicht randomisierten Vergleichs), kann in dieser Datenquelle eine entsprechende Studie ohne Randomisierung mit geänderten Einschlusskriterien (Einschluss von Patientinnen und Patienten mit MTX-Kontraindikationen) durchgeführt werden. Darüber hinaus kann die Beobachtungsdauer über 24 Wochen hinaus erweitert werden.

\section{Anwendungsfeld nicht-medikamentöse Maßnahmen} Beispiel 1

Bei vielen viszeralchirurgischen Eingriffen erfolgt die routinemäßige Einlage einer Drainage, obwohl dieses Thema durchaus kontrovers diskutiert wird. Als Gründe dafür werden das frühe Erkennen von Komplikationen (z.B. Anastomoseninsuffizienzen oder Blutungen) sowie die Ableitung von Flüssigkeit angeführt. Dagegen können die Gefahr möglicher Infektionen, Schmerzen sowie die möglicherweise durch mechanische Irritation selbst ausgelösten Komplika- 
tionen sprechen. Für vergleichsweise unkomplizierte Eingriffe wie Cholezystektomien oder Kolonresektionen existiert zunehmend Evidenz dafür, dass die Einlage einer Drainage keine Vorteile bringt und darauf verzichtet werden kann [27, 28]. Für komplexe Operationen z.B. bei Pankreaseingriffen liegen hingegen vergleichsweise wenige Studien vor. Im Rahmen der PANDRA-Studie wurde die Einlage einer Drainage nach Pankreaskopfresektion in einer RCT gegenüber keiner Einlage verglichen [29]. Primärer Endpunkt der Studie war die Häufigkeit invasiver Reinterventionen während des Krankenhausaufenthaltes. Sekundäre Endpunkte waren u.a. die Krankenhaussterblichkeit, verschiedene Komplikationen sowie Dauer der Operation und des Krankenhausaufenthaltes. All diese Informationen können über elektronische Patientenakten aus dem Krankenhausinformationssystem generiert werden, entsprechend könnten für die oben beschriebene RCT VeDa zur Anwendung kommen.

\section{Beispiel 2}

Für die Behandlung des Rektumkarzinoms in den UICC-Stadien II und III im Bereich des unteren und mittleren Rektumdrittels empfiehlt die aktuelle S3 Leitlinie Kolorektales Karzinom vom Januar 2019 eine neoadjuvante Radiochemotherapie oder Kurzzeit-Radiotherapie [30]. Die zusätzliche präoperative Chemotherapie zur Radiotherapie führt zwangsläufig zu einer höheren Belastung des Patienten, ohne dass bisher Daten aus RCTs zum krankheitsfreien Überleben bzw. zum Gesamtüberleben vorliegen. Um die Frage des Nutzens einer Radiotherapie in Kombination mit einer Chemotherapie zu beantworten, können Daten der klinischen Krebsregister von der Arbeitsgemeinschaft Deutscher Tumorzentren (ADT e.V.) herangezogen werden. Bevor der Einfluss einer neoadjuvanten Radiochemotherapie im Vergleich zur Kurzzeit-Radiotherapie auf das Gesamtüberleben untersucht werden kann, ist hierbei zunächst zu prüfen, inwieweit patientenseitige und zentrumsbezogene Faktoren einen Einfluss auf die Therapieauswahl haben. Gleichzeitig kann darüber abgebildet werden, inwieweit sich die Patienten von denen der bisher vorliegenden RCTs unterscheiden. Aus diesen Informationen könnte anschließend mit den Daten der klinischen Krebsregister eine Target Trial Emulation geplant werden.

\section{Anwendungsfeld komplexe Interventionen}

\section{Beispiel 1}

Antibiotika zählen zu den am häufigsten eingesetzten Arzneimitteln, ein Großteil davon wird von Hausärztinnen und Hausärzten verschrieben. Antibiotikaresistenzen sind ein zunehmendes Public Health Problem. Ziel einer nachhaltigen Versorgung muss es somit sein, unangebrachte Antibiotikaverschreibungen, die am häufigsten bei Infektionen der oberen Atemwege vorkommen, zu reduzieren. Aus verschiedenen Studien ist bekannt, dass Kommunikationstraining sowie Point-of-care Tests (POCT) zu einer Reduktion des unangebrachten Verbrauchs führen können, es fehlen jedoch Langzeitdaten [31]. Im Rahmen einer cluster-randomisierten Studie könnten Hausarztpraxen einer Schulung nebst dem Einsatz von POCT bzw. einer Kontrollgruppe (die bspw. nur Schulungsmaterial erhält) zugeteilt werden. Die Informationen zur Verschreibung von Antibiotika als Endpunkt könnten hier über das Arztinformationssystem gewonnen werden. Gleichzeitig könnten durch eine Verknüpfung mit Daten der Krankenkassen auch weitere Endpunkte wie Hospitalisierungen untersucht werden. Somit könnten auch Daten zur Langzeitwirkung der Intervention generiert werden, ohne dass weiterer Aufwand für die Datenerhebung entsteht.

\section{Beispiel 2}

Gerade im Bereich der Krebsbehandlung können neue Erkenntnisse und Fortschritte die Prognose und Lebensqualität der Betroffenen verbessern. Dabei wird der Kontext der Versorgung in Bezug auf den Behandlungsort sowie die dort vorhandene breite fachliche Erfahrung sowie die Vernetzung und das Qualitätsmanagement immer bedeutsamer. Um eine hohe Behandlungsqualität zu sichern, wurde von der Deutschen Krebsgesellschaft das System der zertifizierten Zentren geschaffen. Die Behandlung in einem zertifizierten Zentrum ist somit eine Intervention von hoher Komplexität, bei der letztlich die Vielzahl der Einzelkomponenten sowie deren Wirkungen zusammenspielen. Für Einzelkomponenten liegen jedoch Wirksamkeitsnachweise aus RCTs vor. Um bspw. belastbare Informationen über die Wirksamkeit der Behandlung in einem Prostatakrebszentrum in Bezug auf Komplikationsraten und Überleben gewinnen zu können, müssen zunächst die Versorgung und deren Ergebnisse sowie die Versorgungsstrukturen der Zentren bzw. der nicht-zertifizierten Kliniken, die diese Operationen ebenfalls erbringen, ermittelt werden. Um alle relevanten Endpunkte und Confounder zu erfassen, können hierfür verschiedene VeDa verknüpft werden. Aus diesen Informationen kann anschließend eine Target Trial Emulation geplant werden.

\section{Beispiel 3}

Die endoprothetische Versorgung von Patienten mit Schenkelhalsfrakturen ist eine operative Intervention bei einem vulnerablen Patientengut. Internationale Publikationen und Analysen von Sozialdaten in Deutschland [32] zeigen, dass rund 25\% der in der Regel betagten und multimorbiden Patientinnen und Patienten das erste Jahr nicht überleben.

Da es sich um Eingriffe der dringlichen Frühversorgung handelt, werden die Patientinnen und Patienten im Allgemeinen durch den Rettungsdienst in das versorgende Haus verbracht. Die Entscheidung, ob Patientinnen und Patienten in einem der rund 500 Endoprothesenzentren (EPZ) [33] oder in einem der über 500 Häuser ohne EPZ behandelt werden, ergibt sich somit eher zufällig. Dies erlaubt, die Behandlung im EPZ als komplexe Intervention zu betrachten.

Mithilfe der Informationen aus dem Endoprothesenregister Deutschland (EPRD) [34] sowie ergänzender Angaben aus den Sozialdaten (z.B. WIdO) kann eine Registerstudie zum Einfluss der Versorgung in einem EPZ auf die Änderung des Pflegegrades, die Morbidität und die Mortalität der Patienten sowie die Standzeiten der Implantate durchgeführt werden.

\section{Ausblick}

Insgesamt legt die Ad hoc Kommission mit diesem zweiten Teil des Manuals einen Rahmen vor für die Anwendung von VeDa zur Evaluation von Interventionseffekten in den Anwendungsfeldern Arzneimitteltherapie, nicht-medikamentöse Maßnahmen und komplexe Interventionen. Die Beispiele wurden vor allem vor dem Hintergrund gewählt, dass die dafür notwendigen Daten zumindest in 
Ansätzen auch für Deutschland vorliegen. Somit verwundert es nicht, dass eine Vielzahl der Beispiele aus der Onkologie stammen, da in diesem Bereich u.a. mit den Krebsregistern hierzulande eine entsprechende Dateninfrastruktur aufgebaut ist.

\section{Weiterentwicklung der Strukturen erforderlich}

Allerdings gibt es trotz bereits gut etablierter Strukturen auch in der Krebsforschung und der Versorgung krebskranker Menschen relevante Defizite in der Vernetzung. Einerseits wird die unter den Bedingungen klinischer Studien nachgewiesene Effizienz innovativer Konzepte nicht systematisch auf ihre Alltagswirksamkeit geprüft. Andererseits werden klinische Daten, die in der Routineversorgung erhoben werden, nur unzureichend für den Erkenntnisgewinn genutzt. Diese Defizite erklären sich durch den Mangel von Vernetzungsstrukturen und eine unzureichend entwickelte versorgungsnahe Krebsforschung.

Um Registerstrukturen und deren Daten nutzen zu können, muss die Verfügbarkeit, Qualität und Aktualität bezüglich der jeweiligen Fragestellung im Vordergrund stehen. Konkret müssen klinische Krebsregister in die Lage versetzt werden, zeitnah vollständige und valide Informationen zu Behandlungen und Endpunkten von den meldenden Ärzten zu erhalten und zu verarbeiten. Zur Nutzung dieser Register für wissenschaftliche Arbeiten mit VeDa müssen Forschungsstrukturen geschaffen werden, die unabdingbar eine Vernetzung zwischen Klinikerinnen und Klinikern, Forscherinnen und Forschern sowie Expertinnen und Experten aus Registern ermöglichen. Diese Strukturen müssen sowohl personell als auch finanziell in den Registern aufgebaut werden. Die Etablierung und Erweiterung der Klinischen Krebsregister um zusätzliche Forschungsbereiche im Verbund mit z.B. Comprehensive Cancer Centern, zertifizierten Zentren, bevölkerungsbezogener Routineversorgung und anderen Versorgungs- und Forschungseinrichtungen beinhalten ein hohes Potenzial. Nur so wird es möglich, bereits bestehende große Datenmengen zu verbinden, zu erweitern und vielfältig zu nutzen.

Gleichzeitig können in Bundesländern, in denen bevölkerungsbezogene Krebsregister die gesetzliche Möglichkeit dazu haben, betroffene Patientinnen und Patienten aus den Registern identifiziert und gezielt über laufende oder geplante RCT informiert werden. Dadurch werden Patientinnen und Patienten bevölkerungsbezogen erreicht - und zwar unabhängig davon, ob sie in einem beteiligten Studienzentrum behandelt werden oder nicht. Voraussetzung dafür ist allerdings, dass die notwendigen Daten und die zugehörigen Auswertungen zukünftig zeitnah zur Verfügung stehen. Dadurch könnte die Repräsentativität erhöht werden, sodass die Ergebnisse besser auf die Gesamtheit der Betroffenen übertragen werden können. Für Fragestellungen, bei denen die Vergleichsgruppe „Care as usual“ erhält, kann zudem durch den Vergleich der Behandlungsverläufe zwischen Teilnehmenden und Nicht-Teilnehmenden besser abgeschätzt werden, ob der in der Studie angewendete Behandlungsstandard sachgerecht ist. Schließlich sollten zukünftig die Krebsregister so aufgestellt werden, dass sie als Datenplattform für die RCTs selbst dienen (registerbasierte RCTs). Dieses Potenzial wird in anderen Ländern bereits intensiver genutzt.

\section{Kostenerstattung im Studienkontext erforderlich}

Eine hohe Hürde für die Durchführung qualitativ hochwertiger Interventionsstudien mit VeDa (z.B. registerbasierte RCTs) stellt potenziell die Finanzierung der Kosten für die zu untersuchenden Interventionen dar. Speziell bei Studien zu Arzneimitteln, bei denen gerade im Hochpreissegment oft Head-to-Head-Vergleiche fehlen, können diese Kosten dazu führen, dass unabhängige Studien allein aus finanziellen Gründen nicht realisierbar sind. Denn obwohl diese Arzneimittel bei zulassungsgemäßem Einsatz außerhalb von Studien von der gesetzlichen Krankenversicherung (GKV) erstattet werden, ist dies bei einem Einsatz in Interventionsstudien nicht der Fall. Hier wäre aus Sicht der Ad hoc Kommission eine gesetzliche Regelung zur Erstattung der Kosten für die zu untersuchenden Interventionen sinnvoll, vorausgesetzt, diese Interventionen (z.B. Arzneimittel) werden in der Versorgung im GKV-System grundsätzlich erstattet, und die geplante Studie ist als qualitativ hochwertig einzuschätzen und beantwortet eine versorgungsrelevante Frage. Hierfür wäre ein entsprechendes Antrags- und Genehmigungsverfahren notwendig. Als Beispiel könnte das etablierte Verfahren beim Gemeinsamen Bundesausschuss (G-BA) zur Finanzierung von Arzneimittelkosten in Studien zur Untersuchung ihrer Anwendung in nicht zugelassenen Anwendungsgebieten dienen (§35c SGB V).

\section{Fazit: Noch viel zu tun}

Damit VeDa regelhaft für die Evaluation von Interventionseffekten nutzbar sind, muss eine entsprechende Dateninfrastruktur sowie eine forschungsfreundliche Auslegung datenschutzrechtlicher Rahmenbedingungen vorliegen. Die Ad hoc Kommission sieht insbesondere im Bereich der Onkologie bereits gute gesetzlich verankerte Voraussetzungen dafür. Langfristig könnte vor allem durch die routinemäßige Etablierung einer systematischen und zeitnahen Erfassung, Aufbereitung und Integration verschiedener komplementärer Datenquellen (z.B. Klinische Krebsregister, GKV-Routinedaten) ein regionales und sektorenübergreifendes Versorgungs- und Qualitätsmonitoring für Tumorerkrankungen in Deutschland geschaffen werden. Mit diesem wäre auch die Evaluation von Interventionseffekten möglich, sei es in registerbasierten RCT oder in Studien ohne Randomisierung in Form einer Target Trial Emulation einschließlich sorgfältiger Korrektur für Confounder, z.B. mittels Propensity Score Matching [35]. Grundsätzlich ist es beim Aufbau weiterer, v. a. mit gesetzlichem Auftrag geschaffener Register erforderlich, diese Möglichkeiten bei der Planung mitzudenken und Kriterien der Eignung bzw. Qualität der Datenerhebung zu berücksichtigen (Tab. 2). Die Eignung der Datenquelle (insbesondere in Bezug auf die Ergebnisqualität und bei Studien ohne Randomisierung auch in Bezug auf das Vorliegen von Informationen zu Baseline bzw. zur Vorgeschichte sowie zu Confoundern) ist die zentrale Voraussetzung für die Durchführung von Studien zur Evaluation von Interventionseffekten auf Basis von VeDa. Die Nutzung von VeDa für Versorgungsanalysen mit einem spezifischen Fokus auf der Qualitätsmessung wird Schwerpunkt einer Folgepublikation sein. 


\section{Interessenkonflikt}

JS nutzt regelmäßig GKV-Routinedaten für wissenschaftliche Forschungsprojekte und ist Leiter des Deutschen Neurodermitisregisters TREATgermany. Er erhielt institutionelle Unterstützung für wissenschaftliche Forschungsprojekte von Sanofi, Novartis, Pfizer und ALK und war als Berater gegen ein Honorar für Sanofi, Novartis, ALK und Lilly tätig. Die anderen Autoren geben keine Interessenkonflikte an.

\section{Literatur}

[1] Pulini AA, Caetano GM, Clautiaux H et al. Impact of Real-World Data on Market Authorization, Reimbursement Decision \& Price Negotiation. Ther Innov Regul Sci 2021; 55: 228-238. doi:10.1007| s43441-020-00208-1

[2] Food and Drug Administration (FDA). Use of real-world evidence to support regulatory decision-making for medical devices. 2017. Im Internet: https://www.fda.gov/media/99447/download

[3] European Medicines Agency (EMA). Guideline on registry-based studies. Draft (24 September 2020, EMA/502388/2020). 2020. Im Internet: https://www.ema.europa.eu/documents/scientific-guideline/ guideline-registry-based-studies_en.pdf

[4] Institut für Qualität und Wirtschaftlichkeit im Gesundheitswesen, (IQWiG). Konzepte zur Generierung versorgungsnaher Daten und deren Auswertung zum Zwecke der Nutzenbewertung von Arzneimitteln nach § 35a SGB V (Auftrag A19-43). 2020. Im Internet: https:// www.iqwig.de/download/\%0AA19-43_Versorgungsnahe-Daten-zumZwecke-der-Nutzenbewertung_\%0ARapid-Report_V1-1.pdf

[5] Li G, Sajobi TT, Menon BK et al. Registry-based randomized controlled trials- what are the advantages, challenges, and areas for future research? J Clin Epidemiol 2016; 80: 16-24 doi:10.1016/j.jclinepi.2016.08.003

[6] Ford I, Norrie J. Pragmatic Trials. N Engl J Med 2016; 375: 454-463. doi:10.1056/nejmra1510059

[7] Schünemann H]. All evidence is real world evidence - The BM] Blog. 2019; https://blogs.bmj.com/bmj/2019/03/29/holger-j-schunemannall-evidence-is-real-world-evidence/Stand: 31.03.2021

[8] Klinkhammer-Schalke M, Kaiser T, Apfelbacher C et al. Manual for Methods and Use of Routine Practice Data for Knowledge Generation. Gesundheitswesen 2020; 82: 717-722. doi:10.1055/a-1237-4011

[9] Schrappe M, Pfaff H. Versorgungsforschung vor neuen Herausforderungen: Konsequenzen für Definition und Konzept. Gesundheitswesen 2016; 78: 689-694. doi:10.1055/s-0042-116230

[10] Kirsch C, Doyle IM, Krause O et al. Lessons learned: Challenges faced in the recruitment process for the cluster-randomized nursing home study 'HIOPP-3 iTBX'. Z Evid Fortbild Qual Gesundhwes 2020; 156-157. doi:10.1016/j.zefq.2020.07.002

[11] Schünemann HJ.. Methodological idiosyncracies, frameworks and challenges of non-pharmaceutical and non-technical treatment interventions. Z Evid Fortbild Qual Gesundhwes 2013; 107: 214-220. doi:10.1016/j.zefq.2013.05.002

[12] Cipriani A, loannidis JPA, Rothwell PM et al. Generating comparative evidence on new drugs and devices after approval. Lancet 2020; 395: 998-1010. doi:10.1016/S0140-6736(19)33177-0

[13] Collins R, Bowman L, Landray M et al. The Magic of Randomization versus the Myth of Real-World Evidence. N Engl J Med 2020; 382: 674-678. doi:10.1056/nejmsb1901642

[14] Hernán MA, Robins JM.. Using Big Data to Emulate a Target Trial When a Randomized Trial Is Not Available. Am J Epidemiol 2016; 183 : 758-764. doi:10.1093/aje/kwv254
[15] García-Albéniz X, Hsu J, Hernán MA. The value of explicitly emulating a target trial when using real world evidence: an application to colorectal cancer screening. Eur J Epidemiol 2017; 32: 495-500. doi:10.1007/s10654-017-0287-2

[16] Sterne JA, Hernán MA, Reeves BC et al. ROBINS-I: A tool for assessing risk of bias in non-randomised studies of interventions. PubMed: BM] 2016; 355: i4919. doi:10.1136/bmj.i4919

[17] Horby PW, Mafham M, Bell JL et al. Lopinavir-ritonavir in patients admitted to hospital with COVID-19 (RECOVERY): a randomised, controlled, open-label, platform trial. Lancet 2020; 396: 1345-1352. doi:10.1016/S0140-6736(20)32013-4

[18] WHO Solidarity Trial Consortium, Pan H, Peto R, et al. Repurposed Antiviral Drugs for Covid-19 - Interim WHO Solidarity Trial Results. N Engl J Med 2020. doi:10.1056/NEJMoa2023184

[19] Bucher HC, Guyatt GH, Griffith LE et al. The results of direct and indirect treatment comparisons in meta-analysis of randomized controlled trials. J Clin Epidemiol 1997; 50: 683-691. doi:10.1016/ S0895-4356(97)00049-8

[20] Rücker G.. Network meta-analysis, electrical networks and graph theory. Res Synth Methods 2012; 3: 312-324. doi:10.1002/jrsm.1058

[21] Janke K, Biester K, Krause D et al. Comparative effectiveness of biological medicines in rheumatoid arthritis: Systematic review and network meta-analysis including aggregate results from reanalysed individual patient data. PubMed: BMJ 2020; 370: m2288. doi:10.1136/ bmj.m2288

[22] Gemeinsamer Bundesausschuss. Nutzenbewertungsverfahren zum Wirkstoff Abemaciclib (Mammakarzinom, HR +, HER2-, Kombination mit Aromatasehemmer). 2019. Im Internet: https://www.g-ba.de/ bewertungsverfahren/nutzenbewertung/410/

[23] Gemeinsamer Bundesausschuss. Nutzenbewertungsverfahren zum Wirkstoff Palbociclib (Mammakarzinom). 2017. Im Internet: https:// www.g-ba.de/bewertungsverfahren/nutzenbewertung/269/

[24] Gemeinsamer Bundesausschuss. Nutzenbewertungsverfahren zum Wirkstoff Ribociclib (Neubewertung nach Fristablauf: Mammakarzinom, HR + , HER2-, Kombination mit Fulvestrant). 2020. Im Internet: https://www.g-ba.de/bewertungsverfahren/nutzenbewertung/527/

[25] Gemeinsamer Bundesausschuss. Nutzenbewertungsverfahren zum Wirkstoff Sarilumab (Rheumatoide Arthritis). 2017. Im Internet: https://www.g-ba.de/bewertungsverfahren/nutzenbewertung/305/

[26] Meissner Y, Richter A, Manger B et al. Serious adverse events and the risk of stroke in patients with rheumatoid arthritis: Results from the German RABBIT cohort. Ann Rheum Dis 2017; 76: 1583-1590. doi:10.1136/annrheumdis-2017-211209

[27] Globke B, Schmelzle M, Bahra M et al. Drainagen in der Viszeralchirurgie: (un)verzichtbar? Chirurg 2017; 88: 395-400. doi:10.1007/s00104-017-0404-5

[28] Cirocchi R, Kwan SH, Popivanov G et al. Routine drain or no drain after laparoscopic cholecystectomy for acute cholecystitis. PubMed noch ahead of print: Surgeon 2020 Jul 24;S1479-666X(20)30057-3. doi: $10.1016 /$ j.surge.2020.04.011. Online ahead of print.

[29] Witzigmann H, Diener MK, Kißenkötter S et al. No need for routine drainage after pancreatic head resection: The dual-center, randomized, controlled PANDRA trial (ISRCTN04937707). Ann Surg 2016; 264: 528-535. doi:10.1097/SLA.0000000000001859

[30] Deutsche Krebsgesellschaft, Deutsche Krebshilfe, AWMF Leitlinienprogramm. S3-Leitlinie Kolorektales Karzinom: Langversion 2.1; 2019

[31] Köchling A, Löffler C, Reinsch S et al. Reduction of antibiotic prescriptions for acute respiratory tract infections in primary care: A systematic review. Implement Sci 2018; 13. doi:10.1186/s13012018-0732-y 
[32] Müller-Mai CM, Schulze Raestrup US, Kostuj T et al. One-year outcomes for proximal femoral fractures: Posthospital analysis of mortality and care levels based on health insurance data. Unfallchirurg 2015; 118: 780-794. doi:10.1007/s00113-013-2534-7

[33] Osmanski-Zenk K, Klinder A, Haas $\mathrm{H}$ et al. Influence of revision surgeries and revision rate on quality indicators in certified centres for joint replacement (endoprothetikzentren). Z Orthop Unfall 2020; 158: 481-489. doi:10.1055/a-0997-6922
[34] Hassenpflug J, Liebs TR. Register als Werkzeug für mehr Endoprothesensicherheit: Erfahrungen aus anderen Ländern und dem Aufbau des Endoprothesenregisters Deutschland. Bundesgesundheitsblatt Gesundheitsforsch - Gesundheitsschutz 2014; 57: 1376-1383. doi:10.1007/s00103-014-2057-6

[35] Agoritsas T, Merglen A, Shah ND et al. Adjusted analyses in studies addressing therapy and harm: Users' guides to the medical literature. JAMA - J Am Med Assoc 2017; 317: 748-759. doi:10.1001/ jama.2016.20029

\section{Hinweis}

Dieser Artikel wurde gemäß des Erratums vom 27.5.2021 geändert.

\section{Erratum}

Im oben genannten Artikel wurde der Name eines Autors

korrigiert. Richtig ist: Karsten Dreinhöfer. 